Abstract IDDF2021-ABS-0121 Table 5 Logistic regression multivariate of risk factors for the degree of acute peripancreatic fluid collections

\begin{tabular}{lllll}
\hline Variable & p-value & B & Exp (B) & $95 \% \mathrm{Cl}$ \\
\hline Calcium & 0.001 & -6.61 & 0.001 & $0.000-0.063$ \\
SIRS $\geq 2$ & 0.026 & 1.34 & 3.819 & $1.177-12.392$ \\
\hline
\end{tabular}

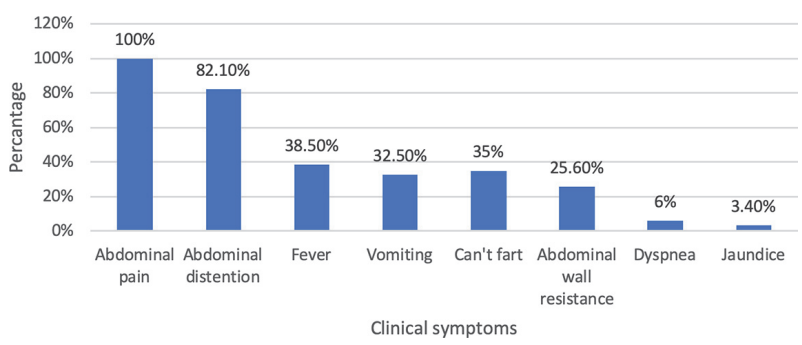

Abstract IDDF2021-ABS-0121 Figure 1

$84.5 \%$, respectively. SIRS score $\geq 2$ in grade $\mathrm{E}$ was significantly higher than in grade D $(89.1 \%$ vs $59.0 \%, \mathrm{p}<0.01)$. (IDDF2021-ABS-0121 Table 3).

Conclusions Alcohol was the most common etiology. Hypocalcemia and SIRS score $\geq 2$ correlated significantly with the occurrence of more acute peripancreatic fluid collections.

\section{IDDF2021-ABS-0124 PREDICTORS OF COMPLICATIONS AND WEIGHT LOSS FOLLOWING INTRA GASTRIC BALLOON INSERTION}

Adnan Alzanbagi*, Abdulaziz Tashkandi, Laeeque Ahmed, Mohammed Khan, Ishtiaque Ahmed, Mahmoud Eliouny, Zaffar Malik, Mohammed Hefny, Mohammed Shariff. King Abdullah Medical City Makkah, Saudi Arabia

\subsection{6/gutjnl-2021-IDDF.146}

Background Obesity is a major global health issue and to address this, multiple weight reduction strategies are emerging. Intragastric balloon (IGB) insertion has shown to be an effective option. However, it is associated with adverse events and weight loss is variable. Hence, the aim of this study is to identify factors associated with weight loss and complications following insertion of IGB.

Methods Retrospective analysis of the complications and weight loss of the patients who had IGB inserted at King Abdullah Medical City, Makkah, a tertiary referral center for Bariatric surgery.

Results Of the 72 patients who had IGB inserted 24 (66.7\%) developed an adverse event, including 5 early removals within 2 weeks. In multivariate stepwise backward logistic regression, female gender was identified as the only predictor of adverse event $(\mathrm{P}=0.009$; odds ratio $0.24795 \%$ confidence interval 0.086 - 0.711). The most common complication was abdominal pain occurring in 15 patients (21\%). Data at 6 months follow-up was available in 67 patients with a mean percentage of excess body weight loss of $17.47 \% \pm 14.21 \%$ with a reduction of BMI of $6.33 \mathrm{~kg} / \mathrm{m}^{2}$. On a multiple regression model, weight loss at 3 months and asthma were significantly correlated with the percentage of excess weight loss $(\mathrm{P}<0.001$ and $\mathrm{P}=0.04$, respectively)
Conclusions In this cohort, the gender of the patients was associated with complications and weight loss at 3 months and asthma predicted the final weight loss.

\section{IDDF2021-ABS-0125 EVIDENCE OF OBJECTIVE ENDOSCOPIC GASTRO ESOPHAGEAL REFLUX POST SLEEVE GASTRECTOMY}

${ }^{1}$ Adnan Alzanbagi*, ${ }^{1}$ Laeeque Ahmed, ${ }^{2}$ Mohammed Alharbi, ${ }^{2}$ Abdulaziz Alzahrani, ${ }^{1}$ Mohammed Khan, 'Zaffar Malik. ${ }^{1}$ King Abdullah Medical City Makkah, Saudi Arabia; ${ }^{2}$ Umm Al Qura University Makkah, Saudi Arabia

\subsection{6/gutjnl-2021-IDDF.147}

Background Sleeve Gastrectomy (SG) is a popular bariatric surgery but its link to gastroesophageal reflux (GERD) is a concern as it's associated with Barrett's esophagus (BE). However, the development of GERD following SG is far from clear. Hence, the aim of this study was to look objectively for endoscopic esophagitis (EE) post SG.

Methods The hospital record of all patients who had post SG endoscopy were reviewed retrospectively. Demographics, comorbidities, medications used, endoscopic findings and helicobacter pylori status were recorded

Results 155 of the 169 patients who had a gastroscopy post SG were included, 14 were excluded, as there were done immediately post SG. Median age of patients was 35 years (range 18-65) with 59\% (92) females and 48\% (74) had hypertension, diabetes mellitus and/or hyperlipidemia. The median post SG endoscopy interval was 16 months (range $7-$ 33). EE was detected in 23\% (36) with $64 \%, 31 \%$ and $5 \%$ having grade $\mathrm{A}, \mathrm{B}$ and $\mathrm{C}$ respectively. None had hiatus hernia and one of them had a $5 \mathrm{~cm}$ Barrett's esophagus (BE). In addition, 26 patients had endoscopy prior to SG of which $15 \%$ (4) had esophagitis post SG and none had esophagitis prior to SG. 19\% (30) were positive for helicobacter pylori (HP) and $23 \%$ of these had esophagitis. Compared to those without HP, the prevalence of esophagitis was not significantly different $(\mathrm{P}$ $=1.00$ ).

Conclusions Endoscopic esophagitis was prevalent in nearly a quarter of our study population. The development of EE may be denovo with the potential to evolve into $\mathrm{BE}$.

\section{IDDF2021-ABS-0127 ASSOCIATION OF HELICOBACTER PYLORI WITH OBESITY IN THE WESTERN POPULATION OF KINGDOM OF SAUDI ARABIA}

${ }^{1}$ Adnan Alzanbagi*, ${ }^{1}$ Laeeque Ahmed, ${ }^{1}$ Sawsan Khan, ${ }^{2}$ Saud Algethami, ${ }^{2}$ Musab Alhumaidi, ${ }^{2}$ Wafaa Alharbi, ${ }^{1}$ Mahmoud Eliouny, ${ }^{1}$ Mohammed Shariff. ${ }^{1}$ King Abdullah Medical City Makkah, Saudi Arabia; ${ }^{2}$ Umm Al Qura University Makkah, Saudi Arabia

\subsection{6/gutjnl-2021-IDDF.148}

Background The prevalence of Helicobacter pylori (HP) in obese patients is conflicting with studies showing a very wide range from $8.7 \%$ to $86 \%$. Hence, the aim of this study was to confirm the prevalence of histologically proven HP in obese patients.

Methods Obese (body mass index (BMI) $\geq 30$, cases) patients who had endoscopy and biopsy for HP in King Abdullah Medical Center (KAMC), Makkah were identified from the 
Abstract IDDF2021-ABS-0127 Table 1 Association of Helicobacter Pylori with obesity in the western Population of kingdom of Suadi Arabia

\begin{tabular}{lll}
\hline BMI & HP Positive (\%) & HP Negative (\%) \\
\hline $30-34$ & 32 & 68 \\
$35-39$ & 29 & 71 \\
$40-44$ & 23 & 77 \\
$50-54$ & 54 & 55 \\
$55-59$ & 50 & 50 \\
$60-64$ & 60 & 40 \\
$>65$ & 73 & 27 \\
\hline
\end{tabular}

hospital information system and their demographics, comorbidities and HP status were recorded retrospective.

Results 468 obese patients (male: female, 1:1.7) with mean age of 48.3 (SD \pm 15 ) years and mean BMI of $44.43 \mathrm{~kg} /$ $\mathrm{m}^{2}$ were analyzed. The prevalence of $\mathrm{HP}$ was $35 \%$ (166/ 469). There was no significant association of HP infection and gender $(\mathrm{P}=0.557)$. There was no difference between the mean ages of patients infected with HP $(40.2 \pm 12$ years) compared to those who were not (42.46 \pm 13.7 years) $(P=0.076)$. Similarly was the case with glycated haemoglobin $(6.79 \pm 1.9$ in HP positive compared with $6.85 \pm 1.7$ in HP negative, $\mathrm{P}=0.708)$. However, the mean BMI was significantly higher in $\mathrm{HP}$ positive patients $\left(46.75 \pm 10.3 \mathrm{~kg} / \mathrm{m}^{2}\right)$ compared to $\mathrm{HP}$ negative (43.1 \pm $\left.8.2 \mathrm{~kg} / \mathrm{m}^{2}\right)(\mathrm{P}=0.0001)$. There was a positive linear correlation with $\mathrm{BMI}$ and $\mathrm{HP}$ prevalence $(\mathrm{P}=0.0007)$ (IDDF2021-ABS-0127 Table 1).

Conclusions The prevalence of HP in our study was positively associated with BMI, rising with increasing BMI. This may have implications for obesity management. Further populationbased studies are needed to confirm.

\section{IDDF2021-ABS-0128 SELF-EXPANDING METALLIC STENT FOR PALLIATION OF MALIGNANT COLORECTAL CANCER: A SINGLE TERTIARY REFERRAL CENTER EXPERIENCE}

${ }^{1}$ Adnan Alzanbagi* ${ }^{1}$ laegue Ahmed, ${ }^{2}$ Mohammed Fatani, ${ }^{2}$ Hussan Alaidarous, IIshtiaque Ahmed, 'Abdulaziz Tashkandi, ${ }^{1}$ Mohammed Khan, ${ }^{1}$ Ali Algesry, ${ }^{1}$ Mohammed Shariff. 'King Abdullah Medical City Makkah, Saudi Arabia; ${ }^{2}$ Umm Al Qura University Makkah, Saudi Arabia

10.1136/gutjnl-2021-IDDF.149

Background 13\% of CRC in the late stage present with intestinal obstruction and there is no clear consensus on how best to manage this clinical emergency. The purpose of this study was to evaluate the effectiveness of Self-expanding metallic stents (SEMS) placement in CRC stenoses in the western region of KSA.

Methods A retrospective review of SEMS placed in King Abdullah Medical City, Makkah, for histological proven inoperable CRC stenosis was done including, demographics data, the success of stent placement, complication, re-intervention and mortality.

Results 26 SEMS were placed in 25 patients. Median age was 51 years (range 37-84) with 64\% (16) males, all CRC had distant metastasis and involved the left side of the colon, except for one located in the hepatic flexure. All SEMS were uncovered with mean length $9 \mathrm{~cm}$. SEMS was placed successfully in all the cases, except one (96\%). Two SEMS placements in sigmoid resulted in perforation requiring emergency surgery, both survived till date. One of the SEMS failed to expand despite appropriate placement ending in colostomy. Tumor in-growth in one case resulted in further obstruction 6 months following SEMS placement. This was successfully treated by restenting with SEMS. There was no SEMS migration or bleeding. Mortality was 52\% (13). Chemotherapy was given to $56 \%$ (14) with one having a perforation. Clinical success of relieving obstruction without complication was achieved in $88 \%$.

Conclusions Placement of SEMS for palliation of obstruction due to CRC in our center was effective in relieving colonic obstruction with complication rates comparable to other studies.

\section{IDDF2021-ABS-0129 METALLIC STENT INSERTION FOR PALLIATION OF ESOPHAGEAL CANCER: SINGLE-CENTRE EXPERIENCE FROM SAUDI ARABIA}

${ }^{1}$ Laeeque Ahmed*, ${ }^{1}$ Adnan Alzanbagi, ${ }^{1}$ Ishtiaque Ahmed, ${ }^{2}$ Bashaer Alzahrani, ${ }^{2}$ Hosam Alghanmi, ${ }^{1}$ Mohammed Shariff. ${ }^{1}$ King Abdullah Medical City Makkah, Saudi Arabia; ${ }^{2}$ Umm Al Qura University Makkah, Saudi Arabia

\subsection{6/gutjpl-2021-IDDF.150}

Background The use of Self-Expandable metallic stents (SEMS) as a non-surgical alternative for palliation of advanced esophageal cancer (EC) is increasing. However, there is a scarcity of real-life experience on the use of these stents exclusively in oesophageal cancer. The aim of this study is to evaluate the efficacy of SEMS in inoperable esophageal cancers in the western region of Saudi Arabia.

Methods A retrospective review of SEMS placed in a tertiary referral hospital for histological proven inoperable EC from 2012 until 2015. Demographics data, procedure success, complication, re-intervention and mortality were analyzed.

Results 46 SEMS were placed in 35 patients for palliation of dysphagia. Median age of patients was 68 years (range 31. 95). 69\% (24) patients have lower third of EC and the rest have middle third (IDDF2021-ABS-0129 Table 1). SEMS was placed successfully in all cases with symptomatic improvement. No major stent-related complication seen. $28 \%$ (13) patients required re-intervention with additional SEMS placement, 9 of which were for tissue in growth and 4 for distal migration. Median survival was 114 days (range 30 - 498). Most of the complications seen in fully covered SEMS compared to the partially covered $50 \%(8 / 16)$ vs $17 \%(5 / 30)$,

Abstract IDDF2021-ABS-0129 Table 1 Characteristics of patients who received SEMS

\begin{tabular}{ll}
\hline Characteristics & $\begin{array}{l}\text { Number }=35 \\
\text { (percentage) }\end{array}$ \\
\hline Age: median (range) & $68(31-95)$ \\
Gender: male: female & $0.8: 1$ \\
Oesophageal Cancer Location Upper Middle Lower & $0(0) 11(31 \%) 24(69 \%)$ \\
Oesophageal cancer type Adenocarcinoma Squamous & $27(77 \%) 8(23 \%)$ \\
carcinoma & $18(51 \%) 17(49 \%)$ \\
Received Chemo/Radiotherapy Yes No & \\
\hline
\end{tabular}

